\title{
Regulatory B cells: The new cells on the block to modulate allergic inflammation.
}

\section{Shawn Khan ${ }^{1} \&$ John-Paul Oliveria ${ }^{1^{*}}$}

1. Department of Medicine, Division of Respirology, McMaster University, Hamilton, ON Canada.

${ }^{*}$ Corresponding author. E-mail: olivejp@mcmaster.ca

Despite its heterogeneity, common clinical manifestations of allergic disease include redness, itchiness and swelling of the affected areas. In those with allergic disease, the exposure to allergens (e.g. antigens that healthy individuals normally have no reaction to, such as pollen and animal dander) can induce IgE-mediated inflammatory processes. The allergic response is canonically mediated by IgE antibodies against allergens, whereby cross-linking of IgE bound on the surface of effector cells propagate the allergic pathways. This can result in the maturation of cluster of differentiation $4^{+}\left(\mathrm{CD4}^{+}\right) \mathrm{T}$ cells into T helper type-2 (Th2) cells and an increase in eosinophilia (1). Eosinophilia, a hallmark of allergic disease manifestation, is the infiltration of the granulocytic cell known as eosinophils, mediated by an increase in Interleukin (IL)-5. Overall, the allergic inflammatory response is facilitated by the release of type 2 cytokines, such as IL-4 and IL-13, which further induce the maturation of IgE-producing B cells (2; Figure 1).

It is thought that the induction of immunological tolerance can mitigate allergic inflammation through the desensitization of the immune system to allergens. Establishing tolerance involves the interplay of regulatory T cells (Tregs), the immunosuppressive IL-10 cytokine, and the process of $\mathrm{T}$ cell anergy where pro-inflammatory responses towards allergic substances are weakened (2).

Currently, there is a renewed interest in B cells as an integral component of both tolerance and the allergic disease framework. While B cells are normally associated with allergy pathogenesis through the production of IgE and other Th2 cytokines, evidence suggests that a subset of B cells (known as regulatory $B$ cells or Bregs) have a regulatory role in suppressing allergen-induced inflammation (2). This review will discuss the inhibitory capacity of Bregs in allergic disease, their mechanism of inhibitory action, and their identification and role in allergen tolerance in human allergies.

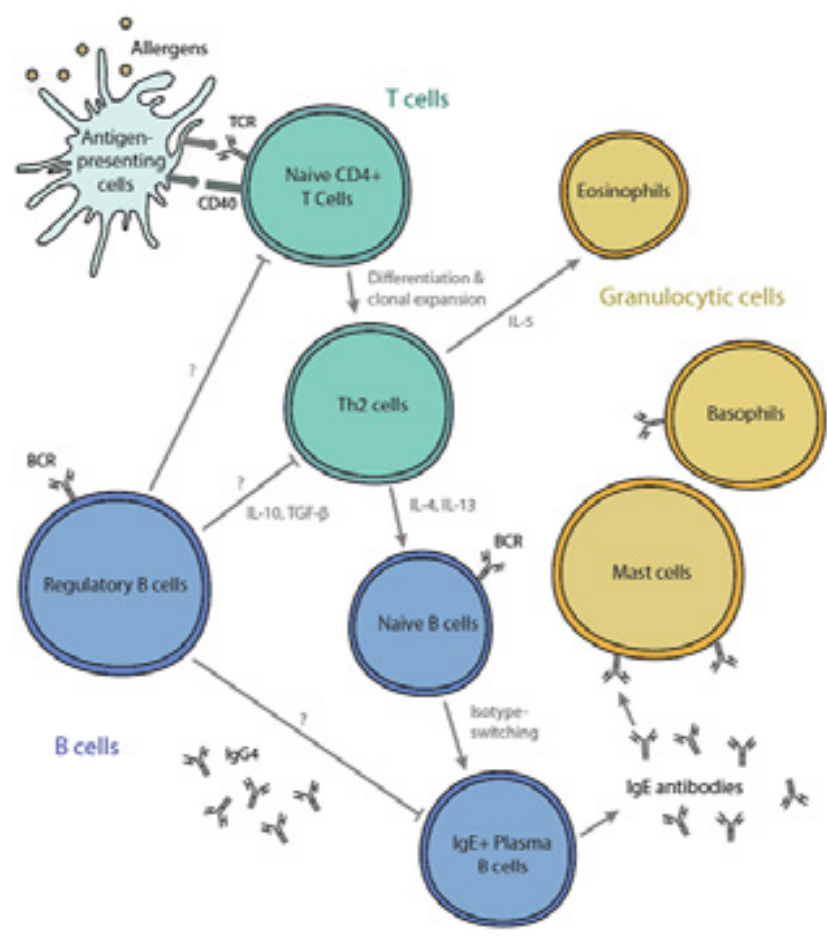

Figure 1. Pathogenesis of the allergic inflammatory cascade in asthma.

\section{The Role of Regulatory B Cells in Allergic Disease}

After the initial characterization of B cells with inhibitory properties by Katz et al. (3), several studies directly showed that the transfer of $B$ cells induced tolerance and attenuated inflammation in mouse models of allergic airway disease, anaphylaxis, and contact hypersensitivity through IL-10 (47). Furthermore, IL-10-producing Bregs have been shown in humans at similar frequencies compared to Bregs in mice (5).

\section{The Mechanistic Function of Regulatory B cells}

Bregs are capable of attenuating inflammation at sites 
of immune activation through the secretion of IL-10 $(\mathrm{Br} 1)$ and TGF- $\beta(\mathrm{Br} 3)$. IL-10 has been shown to have an immunosuppressive effect through the suppression of Th2 inflammatory processes by binding to $T$ cell receptors and blocking co-stimulatory signaling. Meanwhile, TGF- $\beta$ binds $T$ cell receptors to encourage the maturation of Tregs, which have the capacity to inhibit the activation of effector T cells. The disruption of TGF- $\beta$ receptor signaling has been shown to increase an individual's susceptibility to develop allergic asthma. While TGF- $\beta$ has been implicated in mouse allergen tolerance, IL-10 has been implicated in that of humans; however further research needs to be done to determine whether B cell production of TGF- $\beta$ also has allergen tolerance effects in humans. In addition, the inhibitory immunoglobulin, IgG4, is secreted by Bregs to induce a protective effect against IgE by interfering with allergen-lgE interactions and binding to excess allergen (2).

Currently there is interest in the application of Bregs in the field of allergy immunotherapy to achieve an induced state of tolerance and higher levels of IL-10, TGF- $\beta$, and IgG4specific antibodies, thus modulating allergic inflammation (2).

\section{Characterization of Regulatory B cell Phenotypes}

CD19 is considered to be a pan B cell surface marker in both mouse and human models. However, Breg identification is difficult in humans due to a lack of a universal phenotypic characterization. Fortunately, there have been parallels between human and mouse phenotypic characterization of Bregs (5). Common phenotypes in literature used to identify Bregs include: $\mathrm{CD}_{1} \mathrm{~d}^{+} \mathrm{CD}^{+}, \mathrm{CD}^{+} \mathrm{FoxP3}^{+}, \mathrm{CD} 24^{+} \mathrm{CD} 38^{+}$ and $C D 24^{+} C D 27^{+}(5,10-11)$, which have been shown to exert their modulatory role through IL-10 production. Furthermore, it has been shown that these Breg phenotypes express forkhead box 3 (FoxP3), a transcription factor important for regulating the development and function of regulatory $T$ cells. Although the current understanding of Breg development and differentiation into $\mathrm{Br} 1$ and $\mathrm{Br} 3$ is limited due to a lack of mouse and human studies of allergic inflammation (8), several studies are being conducted to elucidate the complexities of Bregs.

In humans it has been shown that levels of $\mathrm{CD}^{+} \mathrm{FoxP3}^{+}$ Bregs were lower in the blood but higher in the airways of allergic asthmatics compared to healthy controls (9-11). These findings were supported by a higher proportion of $\mathrm{IL}-10^{+}$Bregs present in the airways of allergic asthmatics compared to healthy controls (10). Taken together, these findings suggest the possibility that Bregs may be trafficking to sites of inflammation to elicit immunomodulatory processes, however their suppressive roles may not be enough to overcome the chronic allergic inflammation experienced in allergic asthmatics.

\section{Conclusion}

While evidence shows that the primary mode of action of Bregs may occur at local sites of allergic inflammation in an IL-10-dependent manner, TGF- $\beta$ and IgG4 antibodies may also play crucial immunosuppressive roles. Overall, further investigation into the functions and phenotypic identification of regulatory $B$ cells will help build on the complex framework of the pathobiology of allergic disease with the goal of identifying novel drug targets for future therapeutic strategies.

\section{List of abbreviations}

Ag- Antigen, $\mathrm{Br} 1$ - IL-10-producing regulatory $\mathrm{B}$ cell, $\mathrm{Br} 3$ TGF- $\beta$ - producing regulatory $B$ cell, Breg - Regulatory $B$ cell, $C D$ - Cluster of differentiation, FoxP3- forkhead box P3, Ig - Immunoglobulin, IL- Interleukin, TGF- $\beta$ - Transforming Growth Factor $\beta$, Th2 - T helper type-2, Treg - Regulatory T cell

\section{References}

1. Gauvreau GM, Watson RM, O'Byrne PM. Kinetics of allergen-induced airway eosinophilic cytokine production and airway inflammation. Am J Respir Crit Care Med. 1999;160:640-7.

2. Braza F, Chesne J, Castagnet S, Magnan A, Brouard S. Regulatory functions of B cells in allergic diseases. Allergy. 2014;69:1454-63.

3. Katz SI, Parker D, Turk JL. B-cell suppression of delayed hypersensitivity reactions. Nature. 1974;251:550-1.

4. Amu S, Saunders SP, Kronenberg M, Mangan NE, Atzberger A, Fallon PG. Regulatory $B$ cells prevent and reverse allergic airway inflammation via FoxP3-positive T regulatory cells in a murine model. J Allergy Clin Immunol. 2010;125:1114-24.

5. Iwata Y, Matsushita T, Horikawa M, Dilillo DJ, Yanaba K, Venturi GM, et al. Characterization of a rare $\mathrm{IL}-10$-competent B-cell subset in humans that parallels mouse regulatory B10 cells. Blood. 2011;117:530-41.

6. Yanaba K, Bouaziz JD, Matsushita T, Tsubata T, Tedder TF. The development and function of regulatory B cells expressing IL-10 (B10 cells) requires antigen receptor diversity and TLR signals. J Immunol. 2009;182:7459-72.

7. Mangan NE, Fallon RE, Smith P, van Rooijen N, McKenzie AN, Fallon PG. Helminth infection protects mice from anaphylaxis via IL-10-producing $B$ cells. J Immunol. 2004;173:6346-56.

8. Bouaziz JD, Yanaba K, Tedder TF. Regulatory B cells as inhibitors of immune responses and inflammation. Immunol Rev. 2008;224:201-14.

9. Oliveria JP, Beaudin S, Watson R, Campbell H, Howie K, Scime T, et al. Circulating levels of regulatory B cells in asthmatic subjects. Am J Respir Crit Care Med. 2014;189:A3686.

10. Oliveria JP, Tenn M, Smith S, Baatjes A, Obminski C, Scime T, et al. Detection of regulatory B cells in the airways of subjects with asthma. Am J Respir Crit Care Med. 2015;191:A4186.

11. Oliveria JP, Phan S, Tenn MW, Watson BM, El-Gammal Al, Chen R, et al. 
Assessing the expression of FoxP3 on different phenotypes of circulating regulatory B cells in mild asthmatic subjects. Am J Respir Crit Care Med. 2015;191:A4156.

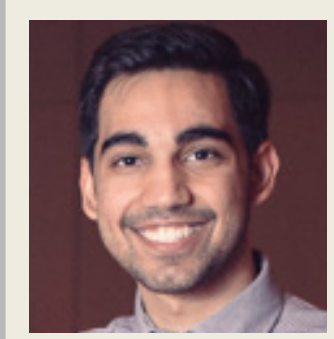

\section{Shawn Khan}

Shawn Khan is a 2nd year undergraduate student in the Bachelor of Health Sciences program specializing in Global Health at McMaster University. He began volunteering at the McMaster Cardio-Respiratory Lab in 2015 under the supervision of Dr. Gail Gauvreau and John-Paul Oliveria. Currently, he is doing his research project with the lab to explore the regulatory capacity of $B$ cells in the context of allergic disease.

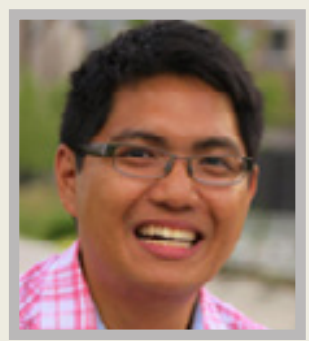

\section{John-Paul Oliveria}

John-Paul Oliveria is a 3rd-year Doctor of Philosophy Candidate in the Department of Medical Sciences at McMaster University. He began his MSc in the McMaster CardioRespiratory Lab in 2011 and transferred to his PhD in 2013. His research focuses on the biology of $B$ cells (IgE+ B cells and regulatory $B$ cells) and the pathogenesis of allergic asthma. 